\title{
Electrochemical detection of phenolic compounds using tyrosinase modified glassy carbon electrode
}

\author{
A. Maikap, K. Mukherjee, N.Mandal and B. Mondal ${ }^{*}$
}
Centre for Advanced Materials Processing, CSIR-Central Mechanical Engineering Research Institute, Durgapur-713209, W.B. India
*Email: bnmondal@rediffmail.com

Phenolic contaminants are now great threat for living organism due to their protein-degenerating effect. The phenolic derivatives are considered as major organic pollutants present in drinking water of various terraces of the world. Sensitive detection of such phenolic contaminants using simple and cost effective sensors is demanding. In the present study, glassy carbon electrodes (GCE) and their tyrosinase modified counterparts (GCTE) are used to prepare working electrodes for the electrochemical detection of $10 \mathrm{ppm}$ catechol. Tyrosinase, an enzyme, facilitates the oxidation of catechol to o-quinone which enhances the electron mobility. As a result, the GCTE powders are found more sensitive than GCE for the detection of catechol.

The electrochemical behaviour of Tyrosinase modified glassy carbon paste electrode (GCTE) is appraised in comparison to that of GCE. Important shifting in the peak potentials and increases in the peak currents is indicative for the development of biosensor for detecting phenolic contaminants. The performance of the biosensor has been evaluated by studying the parameters of cyclic voltammetry (i.e., current peak, $\Delta \mathrm{Ep}$, anodic and catholic current ratio, charge density) and the amperometric response to catechol.

It has been observed from cyclic voltametric measurement of GCTE and GCE that the reduction potential of 0.014 volt with 2 electron passes per ion occur in case GCTE wheras the reduction potential of 0.0324 volt with 1 electron passes per ion occur in case of GCE as shown in Figure 1(a). It has also been observed that the magnitude of cathodic and anodic current of GCTE are significantly greater than GCE.

The impedance spectroscopy of both the electrodes reveal the transportation phenomenon of electron following simplified Randles cell models. The equivalent circuit of both the electrodes is depicted in the inset of Figure (b). High resolution microscopic image is cleraly shown that succesful immobilization of enzyme (Tyrosinase) with glassy carbon takes place as shown in Figure (c).
Amperometric study has been carried out at $-3.8 \mathrm{~V}$ (from the $\mathrm{CV}$ electrocatalysis) for biosensing application and the $\mathrm{N}_{2}$ saturated PBS ( $\mathrm{pH} 7$ ), stirred at $1200 \mathrm{rpm}$ exhibits fast and well defined response towards each catechol additions (Figure d). The response current increases linearly in the range of $1.0-22.48 \mu \mathrm{M}$ with a sensitivity of 15.49 $\mu \mathrm{A} \mu \mathrm{M}^{-1} \mathrm{~cm}^{-2}$. The stability of GCTE is evident at $0.02 \mu \mathrm{A}$ current. Once the stability is reached, $50 \mu \mathrm{M}$ of catechol is added as the analyte. Then the current increases with small response time and becomes constant after a short interval of time which indicates that the developed electrode has a excellent catalytic activity towards cathechol sensing.

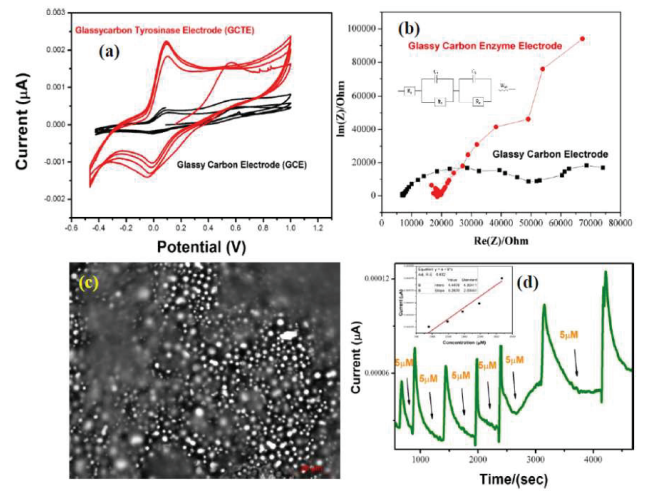

Figure 1: (a) Cyclic Voltammetry of GCTE \& GCE (Red \& Black respectively); (b) Impedance Spectra of GCTE \& GCE (Red \& Black respectively); (c) Microscopic image of GCTE surface; (d) Amperometric study and typical calibration graph of the biosensor for phenol.

\section{References}

1. Abdullah, J., Ahmada, M., Karuppiah, N., Henga, L.Y., Sidek, H., Sensors and Actuators B 114, 2006 604-609.

2. Krishnaraj, N. R., Karthikeyan, R., Berchmans, S., Chandran, S., Pal, P., Electrochimica Acta 112, 2013 465472. 